\title{
ATTENTIONAL TRADE-OFFS UNDER RESOURCE SCARCITY
}

by

Brandon M. Tomm

B.A. (Hons) The University of the Fraser Valley, 2014

A THESIS SUBMITTED IN PARTIAL FULFILLMENT OF THE REQUIREMENTS FOR THE DEGREE OF

MASTER OF ARTS

in

The Faculty of Graduate and Postdoctoral Studies

(Psychology)

THE UNIVERSITY OF BRITISH COLUMBIA

(Vancouver)

August, 2017

C Brandon M. Tomm, 2017 


\begin{abstract}
Resource scarcity poses challenging demands on the cognitive system. Budgeting with limited resources induces an attentional focus on the problem at hand, but it also comes with a cost. Specifically, scarcity causes a failure to notice beneficial information in the environment, or remember to execute actions in the future, that help alleviate the condition of scarcity. This neglect may arise as a result of attentional narrowing. Attentional trade-offs under scarcity can further determine memory encoding. In seven experiments, we demonstrated that participants under scarcity prioritized price information but neglected a useful discount when ordering food from a menu (Experiment 1); they showed better recall for information relevant to the focal task at a subsequent surprise memory test (Experiments 2 and 3); they performed more efficiently on the focal task but neglect a useful cue in the environment that could save them resources (Experiments 4-6); and they failed to remember the previous instructions to execute future actions that could save them resources (Experiment 7). These results collectively demonstrate that scarcity fundamentally shapes the way people process information in the environment, by directing attention to the most urgent task, while inducing a neglect of other information that can be beneficial. The attentional neglect and memory failures may lead to suboptimal behaviors that further aggravate the condition of scarcity. The results provide new insights on the behaviors of the poor, and also important implications for public policy and the design of welfare services and programs for low-income individuals.
\end{abstract}




\section{Lay Summary}

Scarcity is the condition of having insufficient resources (e.g., money, time), which presents significant challenges to the cognitive system. For example, scarcity directly impairs intelligence and can cause myopic behaviours. This neglect may arise as a result of attentional narrowing. We propose that scarcity shapes the way people process information in the environment by directing attention to the most urgent task, while inducing a neglect of other information that can be beneficial. In seven laboratory experiments, we demonstrate how scarcity causes people to prioritize task-relevant information, which facilitates memory performance, but also comes at the expense of neglecting other useful information in the environment. Ironically, this neglected information can help alleviate the condition of scarcity itself. The results provide new insights on the behaviors of the poor, and important implications for public policy and the design of welfare services and programs for low-income individuals. 


\section{Preface}

These studies were conducted at the Behavioral Sustainability Lab at the University of British Columbia. All experimental design, data collection, and data analysis was conducted by the lead author, Brandon M. Tomm, under the supervision of Dr. Jiaying Zhao. Data was collected with the help of research assistants at the Behavioral Sustainability Lab.

Parts of Chapter 1 are currently under review. Chapters 2 and 3 are published in the Proceedings of the Cognitive Science Society (Tomm \& Zhao, 2016). Chapters 4 and 5 have been accepted in the Proceedings of the Cognitive Science Society this year (Tomm \& Zhao, in press). This thesis will be submitted as a manuscript to a journal for peer review.

All experiments reported here were approved by the UBC Behavioral Research Ethics Board (H13-02686). 


\section{Table of Contents}

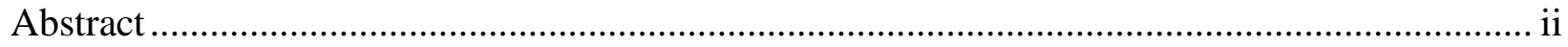

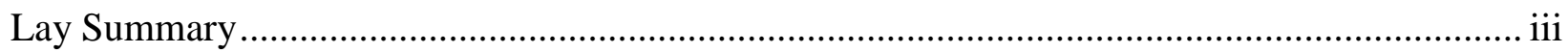

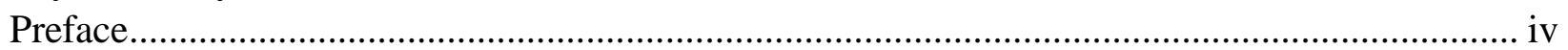

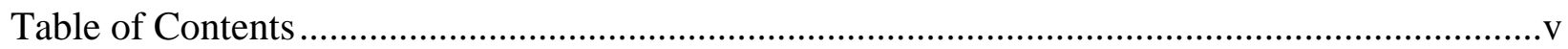

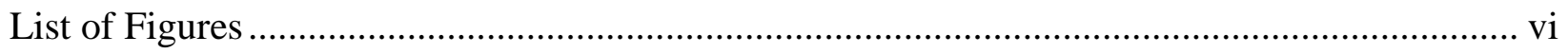

Acknowledgements ........................................................................................................ vii

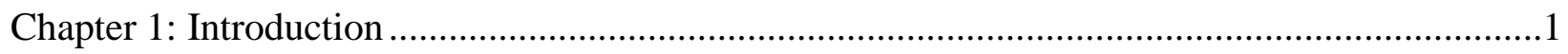

Chapter 2: Attentional Focus and Neglect under Scarcity ............................................................5

2.1 Experiment 1 ..................................................................................................

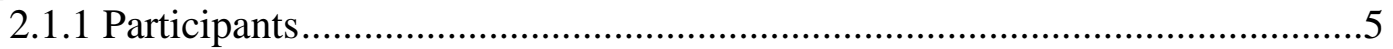

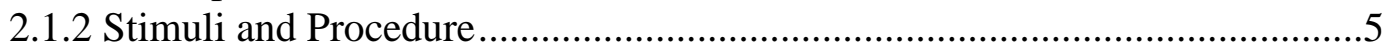

2.1.3 Results and Discussion ............................................................................

Chapter 3: Memory Enhancement of Scarcity-relevant Information .............................................12

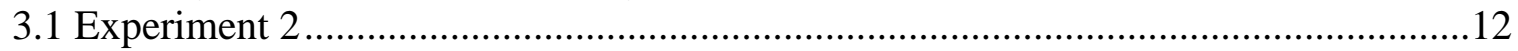

3.1.1 Participants....................................................................................12

3.1.2 Stimuli and Procedure ..............................................................................12

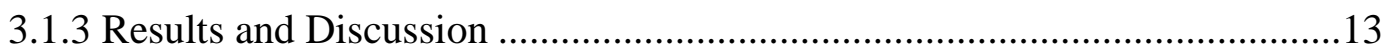

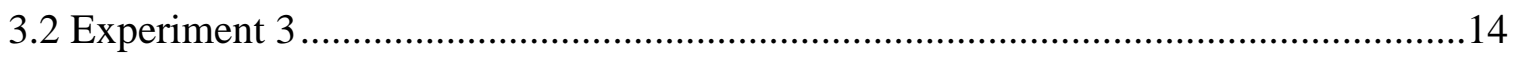

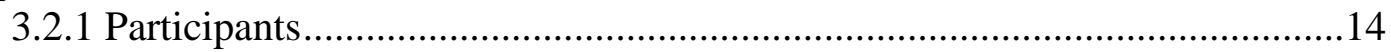

3.2.2 Stimuli and Procedure............................................................................14

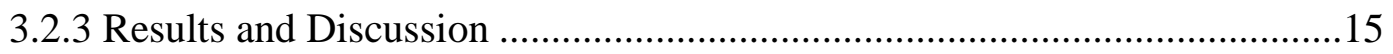

Chapter 4: Spatial Narrowing of Attention under Scarcity.........................................................17

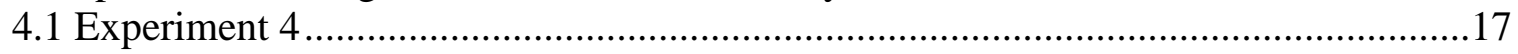

4.1.1 Participants......................................................................................17

4.1.2 Stimuli and Procedure ..............................................................................17

4.1.3 Results and Discussion ........................................................................19

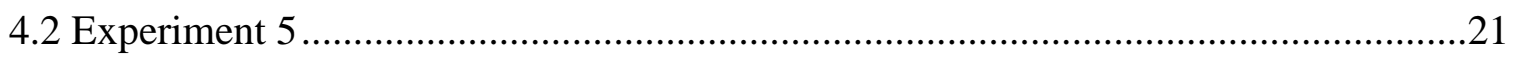

4.2.1 Participants, Stimuli, and Procedure ......................................................21

4.2.2 Results and Discussion ........................................................................22

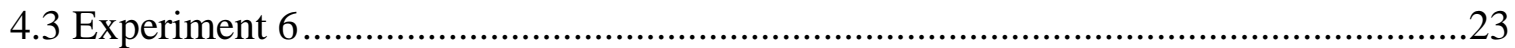

4.3.1 Participants, Stimuli, and Procedure .............................................................23

4.3.2 Results and Discussion .........................................................................24

Chapter 5: Prospective Memory Failures under Scarcity ……………………….....................26

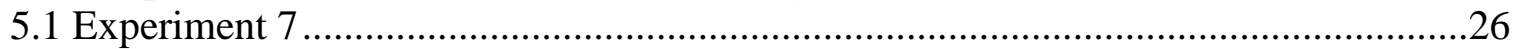

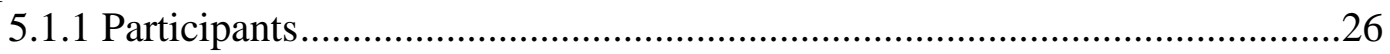

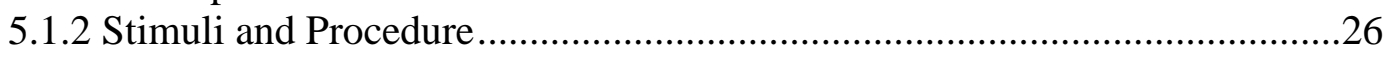

5.1.3 Results and Discussion ..........................................................................27

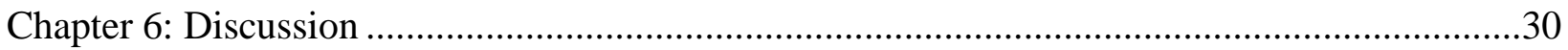

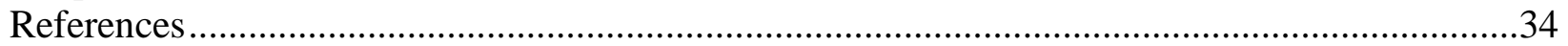




\section{List of Figures}

Fig. 1.1: Theoretical model on the vicious cycle of scarcity (Introduction) ................................3

Fig. 2.1: Heat map visualization of eyetracking data (Experiment 1) ........................................6

Fig. 2.2: Dwell time and Fixations on food items (Experiment 1) ...........................................

Fig. 2.3: Dwell time and Fixations on prices (Experiment 1) ...................................................9

Fig. 2.4:Dwell time and Fixations on calories (Experiment 1) .............................................

Fig. 2.5:Dwell time and Fixations on the discount clause (Experiment 1) ................................10

Fig. 3.1: Memory recall error for prices and calories (Experiment 2) .....................................13

Fig. 3.2: Memory recall error for prices and calories (Experiment 3) ....................................15

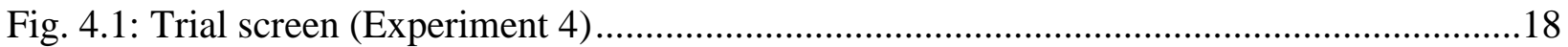

Fig. 4.2: Raven's performance and cue detection results (Experiment 4) ...............................20

Fig. 4.3: Trial screen (Experiment 5) ....................................................................................22

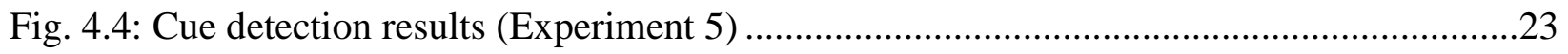

Fig. 4.5: Trial screen (Experiment 6) ...................................................................................24

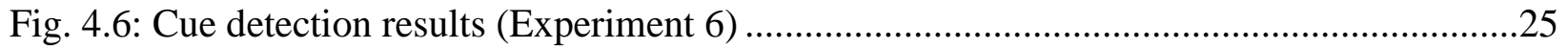

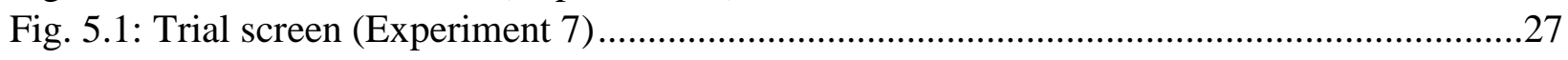

Fig. 5.2: Raven's performance and prospective memory performance results (Experiment 7) ....29 


\section{Acknowledgements}

I would like to thank the grand host of students and research assistants who helped the research process by collecting data, offering suggestions, and making the lab a wonderful place to work: Bram Scheerlinck, Carly Chui, Desmond Fung, Ivy Tam, Justin Reed, Lauren Duda, Mark Ng Shun, May Wang, Patricia Sun, Sherry Guo, Yimiao Gong, and Vincent Wang. Many thanks also to the faculty members and fellow graduate students who gave their thoughtful feedback. Most of all, I would like to thank Dr. Jiaying Zhao for her great mentorship which has profoundly shaped my scientific approach. 
Chapter 1: Introduction

Scarcity is an urgent and pervasive problem in the world: Roughly 1.2 billion people live without electricity (International Energy Agency, 2016), 663 million people lack access to clean water (UNICEF \& World Health Organization, 2015), and 10.7\% of the world population live with less than US\$1.90 per day (World Bank, 2016). Scarcity is the condition of having insufficient resources to cope with demands, and presents significant challenges to the cognitive system. With regard to finances, having a small budget requires the meticulous calculation of current and upcoming expenses, and juggling of sporadic incomes. For many people on a daily basis, the shortage of time also requires management of complicated schedules. Regardless of the resource domain, scarcity imposes considerable demands on the human cognitive system.

Some cognitive consequences of scarcity are recently revealed by a number of lab and field studies (Mullainathan \& Shafir, 2013). For example, scarcity causes myopic behavior which results in the neglect of future events (Shah, Mullainathan, \& Shafir, 2012). Specifically, people under scarcity tend to prioritize the task at hand but over-borrow resources from the future. Financial scarcity directly impairs cognitive function, reducing fluid intelligence and the ability to exert cognitive control (Mani, Mullainathan, Shafir, \& Zhao, 2013). These cognitive and behavioral consequences are particularly problematic because these impairments can lead to suboptimal decision making and behaviors (e.g., poor time management or financial planning skills) that further perpetuate the condition of scarcity. To this end, the study of psychological responses to scarcity can help shed light on the larger question: why do the poor stay poor?

A primary function of scarcity is drawing attentional focus to the task at hand. For example, people who are hungry are more likely to detect food-related cues on a computer screen compared to people who have recently eaten (Piech, Pastorino, \& Zald, 2010; Radel \& Clément- 
Guillotin, 2012). People who are thirsty are more likely to focus on water-related cues (Aarts, Dijksterhuis, \& De Vries, 2001). Alcoholics and dieters are more likely to attend to alcohol and food-related cues, respectively (Stetter, Ackermann, Bizer, Straube, \& Mann, 1995). People with retirement or financial anxiety attend more readily to retirement or money-related cues (Gutierrez \& Hershey, 2013; Shapiro \& Burchell, 2012), and low-income people are more likely to know the starting price of a taxi than high-income people, despite how the poor take taxis less frequently than the rich (Mullainathan \& Shafir, 2013). These examples represent initial evidence that scarcity prioritizes information in the specific domain of scarcity.

Currently, it is still unclear what cognitive mechanisms underlie the impairments caused by scarcity. A possible explanation is that scarcity presents urgent demands that hijack attentional resources. The urgent demands cause a strong focus on the task at hand which can induce a neglect of other potentially important information. Support for this explanation comes from the previous theoretical and empirical work on the limits of the cognitive system. Specifically, the cognitive system has a finite capacity, and people can only receive and process a limited amount of information at a time (Baddeley, 1992; Luck \& Vogel, 1997; Miller, 1956; Pashler, Johnston, \& Ruthruff, 2001). Given this limited capacity, engaging in one process consumes cognitive resources which may be needed for another, thus causing interference. For example, studies on inattentional blindness (Simons \& Chabris, 1999) show that performing a demanding task (e.g., counting how often the basketball is passed around) results in an inability to notice a salient event (e.g., a man dressed as a gorilla passing by). Basic visual features of unattended stimuli may not even be perceived (Rock \& Gutman, 1981). In addition to perception, this interference can cause serious behavioral consequences such as impaired driving (Strayer, Drews, \& Johnston, 2003). The limited cognitive resources given competing demands 
can thus result in attentional trade-offs between focus and neglect. We propose that scarcity causes attentional demands which cause neglect in the same way.

Importantly, this cognitive account under scarcity is distinct from other common explanations on poverty. Instead of implicating personal factors such as education (Bernheim, Garrett, \& Maki, 2001), or personality traits (Salling \& Harvey, 1981), the present work emphasizes that the condition of scarcity itself impacts behaviour. The people themselves living under poverty need not be especially deviant from the average person, but the environmental conditions of scarcity can shape their behaviour. A key message of the recent work is that the poor are often met with inappropriate character assassinations which fail to account for the cognitive consequences of their prevailing circumstances (see Bertrand, Mullainathan, \& Shafir, 2004).

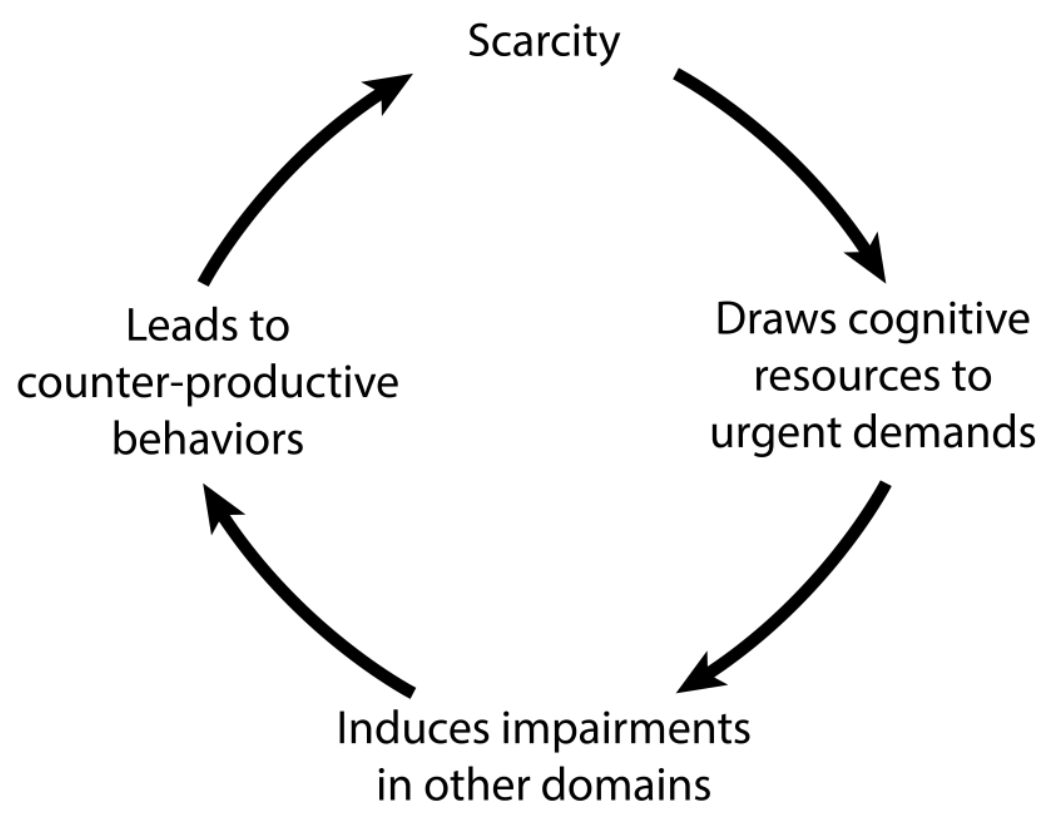

Fig. 1.1. Theoretical framework illustrating the vicious cycle of scarcity.

In the present work, we propose that scarcity forces attentional trade-offs. Specifically, people operating under scarcity may prioritize urgent tasks at hand, leaving other information 
unattended. This process can be counter-productive because the attentional neglect can cause the failure to notice other information in the environment. The neglected information can be beneficial to the poor, and could help alleviate the condition of scarcity. Failures to notice beneficial information can perpetuate a vicious cycle of scarcity (see Figure 1.1). We conducted empirical work demonstrating how scarcity causes cognitive trade-offs which can enhance performance where it is most needed, but also cause cognitive and behavioural neglect.

To investigate the attentional trade-offs under scarcity and the resulting cognitive and behavioural consequences of such trade-offs, we conducted seven experiments in the current study. First, we demonstrate that participants under financial scarcity prioritized price information but neglected a useful discount when ordering food from a menu (Experiment 1). Next, participants under scarcity showed selectively facilitated memory recall for information relevant to the focal task relative to other information at a subsequent surprise memory test (Experiments 2 and 3). Under the condition of time scarcity, we demonstrate that participants performed more efficiently on the focal task but neglect a useful cue in the environment that could save them resources (Experiments 4-6). Finally, we demonstrate how participants under time scarcity failed to remember instructions to execute future actions which could save them resources (Experiment 7). The experiments reported in this thesis demonstrate that scarcity fundamentally shapes the way people process information in the environment, by directing attention to the most urgent task, while inducing a neglect of other information that can be beneficial. The attentional neglect and memory failures may lead to suboptimal behaviors that further aggravate the condition of scarcity. The results provide new insights on the behaviors of the poor, and also important implications for public policy and the design of welfare services and programs for low-income individuals. 
Chapter 2. Attentional Focus and Neglect under Scarcity

\subsection{Experiment 1}

The goal of this experiment was to examine the effects of scarcity on visual attention. We predict that scarcity draws attention to the information relevant to the task at hand, but at the same time, also causes the neglect of other useful information in the environment.

\subsubsection{Participants}

One hundred and ninety undergraduate students (152 female, 35 male, 3 unspecified; mean age $=20.39$ years, $\mathrm{SD}=3.92$ ) were recruited from the Human Subject Pool at the Department of Psychology at the University of British Columbia (UBC), and participated in the experiment for course credit. Participants in all experiments reported normal or corrected-tonormal vision and provided informed consent.

\subsubsection{Stimuli and Procedure}

Participants were presented with a restaurant menu which contained 24 food items. For each item, the price and the calories were listed in two columns on the menu (Figure 2.1). The menu subtended $12.4^{\circ}$ of visual angle in width and $16.2^{\circ}$ in height. A discount clause was shown on the bottom of the menu ("You may ask for an 18\% student discount.").

Participants were randomly assigned with either a small budget ( $\$ 20$; the poor condition) or a large budget ( $\$ 100$; the rich condition). Thus, the experiment used a between-subjects design. Participants were asked to view the items on the menu and think about what they would like to order, as if they were ordering a meal from a restaurant. They were given unlimited time to place the order, and were told not to exceed the assigned budget, but they were not required to 
spend the entire budget. When participants had decided what they would like to order, they reported the items verbally to the experimenter.

The eye gaze of each participant was monitored throughout the experiment using SMI RED-250 Mobile Eyetracking System (60hz). To examine which part of the menu was attended to, the menu was divided into four areas of interest: food items (left column), price information (middle column), calorie information (right column), and discount clause (on the bottom).
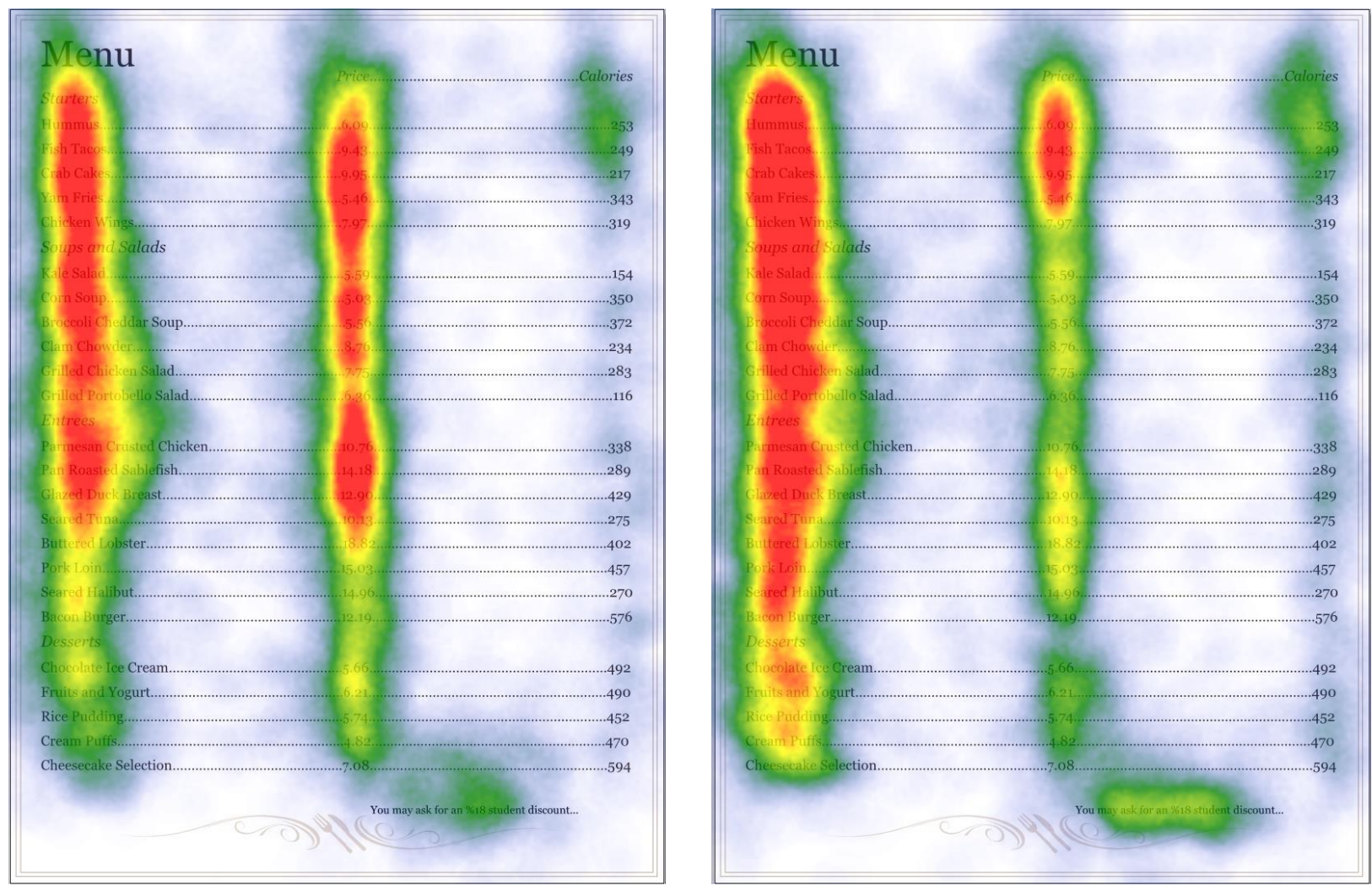

Fig. 2.1. Left: A heat map of the menu showing the distribution of the average dwell time for the participants in the poor condition (who ordered a meal with \$20). Right: A heat map showing the distribution of the average dwell time for the participants in the rich condition (who ordered a meal with \$100). Warmer colors represent longer average dwell time. 


\subsubsection{Results and Discussion}

To measure visual attention, we calculated the dwell time and the number of fixations in each area of interest. The heat maps of the average duration of dwell time between the poor and the rich conditions were shown in Figure 2.1.

Since there was no time limit in the experiment, participants could spend as much time as they needed to make the order. We found that participants in the rich condition took more time to order $(\mathrm{M}=89$ seconds $)$ than the participants in the poor condition $(M=76$ seconds $)[t(188)=$ $2.24, p=.03, d=.33]$. Thus, we used the proportional dwell time (the dwell time spent in each area divided by the total dwell time on the menu) and the proportional fixations (the number of fixations in each area divided by the total number of fixations on the menu) as two measures of visual attention. In addition, participants with more than 3 standard deviations away from the mean in each measure were excluded (between 1 and 4 participants in total, depending on the measure).

For the food items (Figure 2.2), participants in the poor condition spent less dwell time $(M=35.11 \%)$ than those in the rich condition $(M=51.98 \%)[t(185)=3.91, p<.001, d=.57]$. The poor also made few fixations $(M=36.66 \%)$ on the food items than the rich $(M=48.50 \%)$ $[t(187)=3.08, p=.002, d=.45]$. This suggests that the poor participants spent less time considering which food items they would like to order than the rich participants did.

For price information (Figure 2.3), participants in the poor condition dwelled longer at prices $(M=21.08 \%)$ than those in the rich condition $(M=15.23 \%)[t(185)=2.16, p=.03, d$ $=.32]$. Participants in the poor condition also made more fixations on prices $(M=23.07 \%)$ than 
those in the rich condition $(M=15.81 \%)[t(185)=2.91, p<.01, d=.43]$. This suggests that the poor attended more to prices than the rich participants.
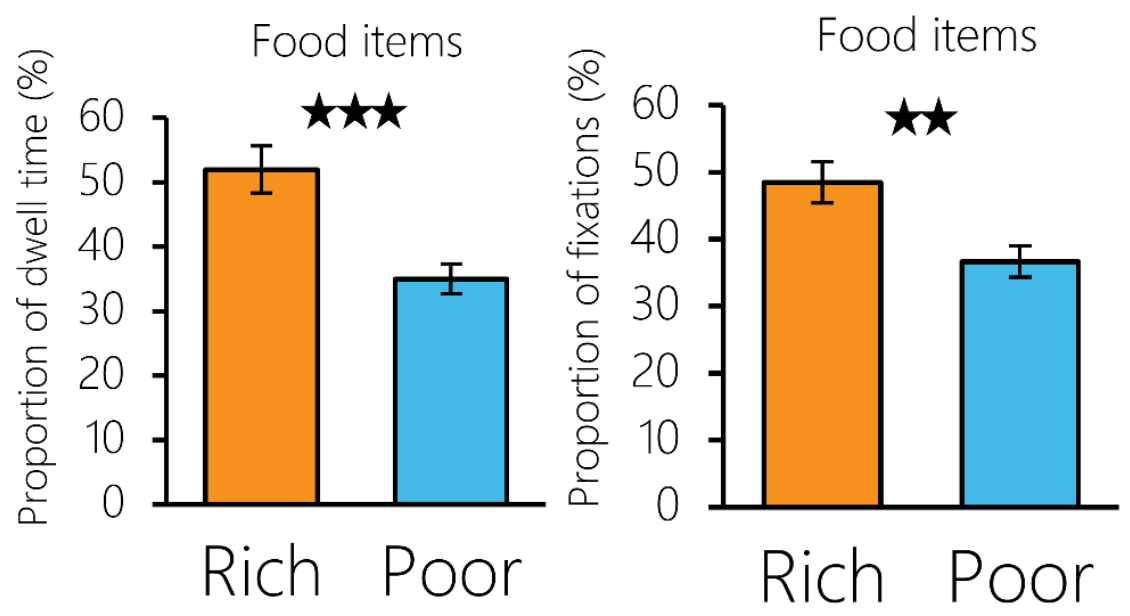

Fig. 2.2. The proportional dwell time and fixations on food items between participants in the poor and the rich conditions (error bars reflect \pm 1 SEM; **p<.01, ***p<.001).

This result could be driven by the possibility that scarcity enhanced attention to all numerical information. Thus, we examined attention to the calorie information (Figure 2.4). Participants in the poor condition dwelled less on calories $(M=2.92 \%)$ than those in the rich condition $(M=4.35 \%)[t(185)=2.65, p<.01, d=.39]$. The poor $(M=3.51 \%)$ also made fewer fixations on calories than the $\operatorname{rich} \operatorname{did}(M=5.09 \%)[t(184)=2.39, p=.02, d=.35]$. This indicates that financial scarcity draws attention more to prices and induces a neglect of calories. 

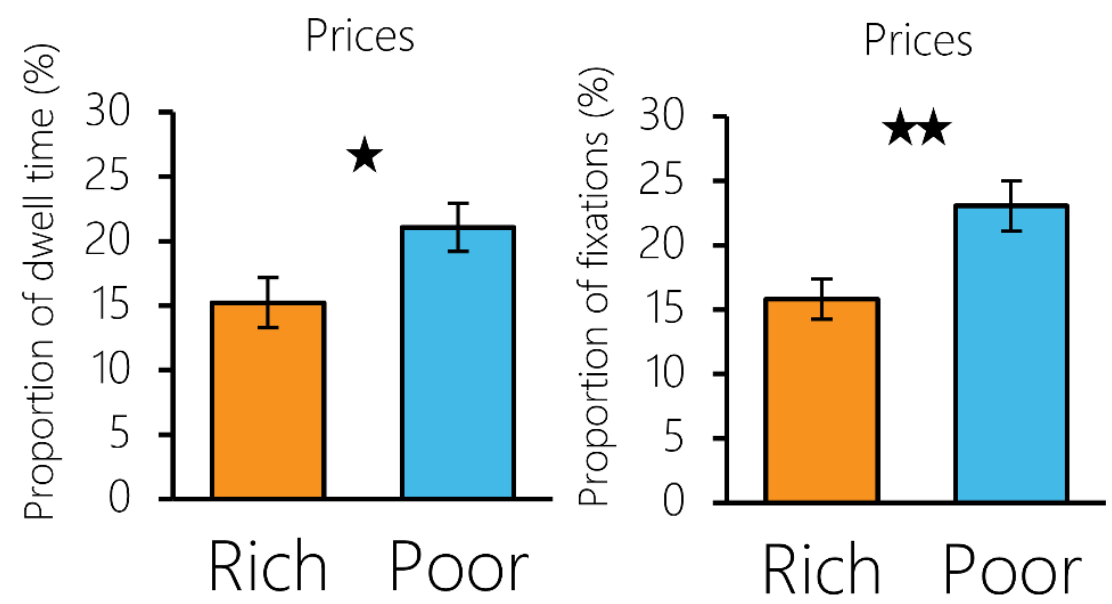

Fig. 2.3. The proportional dwell time and fixations on prices between participants in the poor and the rich conditions (error bars reflect \pm 1 SEM; *p<.05, **p<.01).
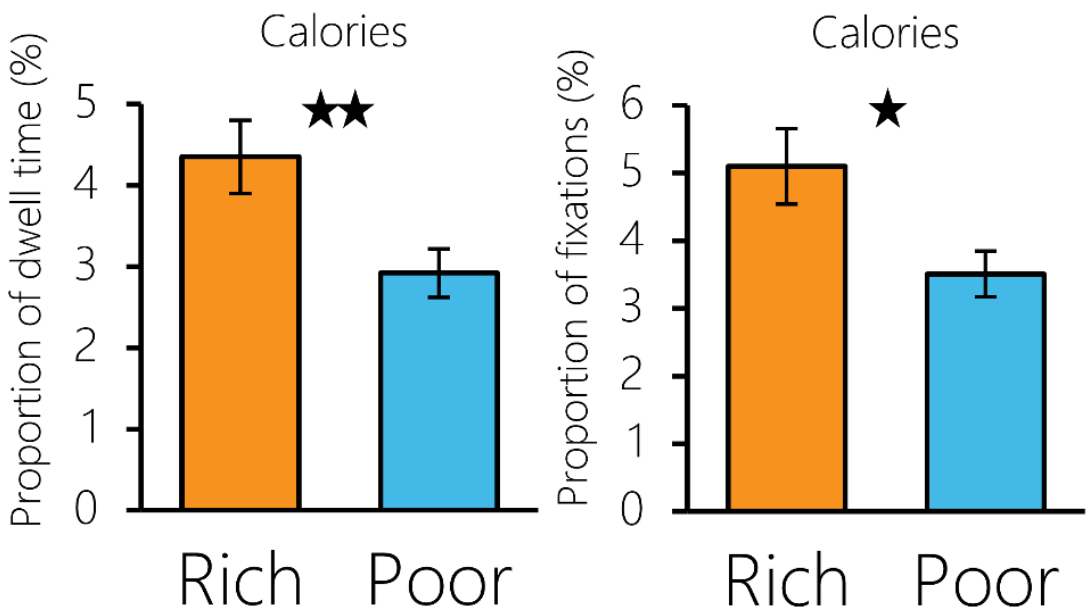

Fig. 2.4. The proportional dwell time and fixations on calories between participants in the poor and the rich conditions (error bars reflect \pm 1 SEM; $* p<.05, * * p<.01$ ).

Importantly, for the discount clause (Figure 2.5), participants in the poor condition spent less dwell time $(M=0.83 \%)$ than those in the rich condition $(M=1.83 \%)[t(184)=3.51, p$ $<.001, d=.52]$. The poor also made fewer fixations on the discount clause $(M=0.85 \%)$ than the $\operatorname{rich}(M=1.82 \%)[t(184)=3.51, p<.001, d=.52]$. This suggests that the poor neglected the discount, compared to the rich participants. 
As another measure of attention to the discount, after placing the order the participants were asked if they had noticed other information on the menu besides the price and calorie information. While measures of visual attention showed that the poor looked less at the discount than the rich did, there was no reliable difference between the number of poor $(N=36)$ and rich $(N=33)$ participants who explicitly reported noticing the discount $\left[X^{2}(1,190)=.09, p=.76\right]$.
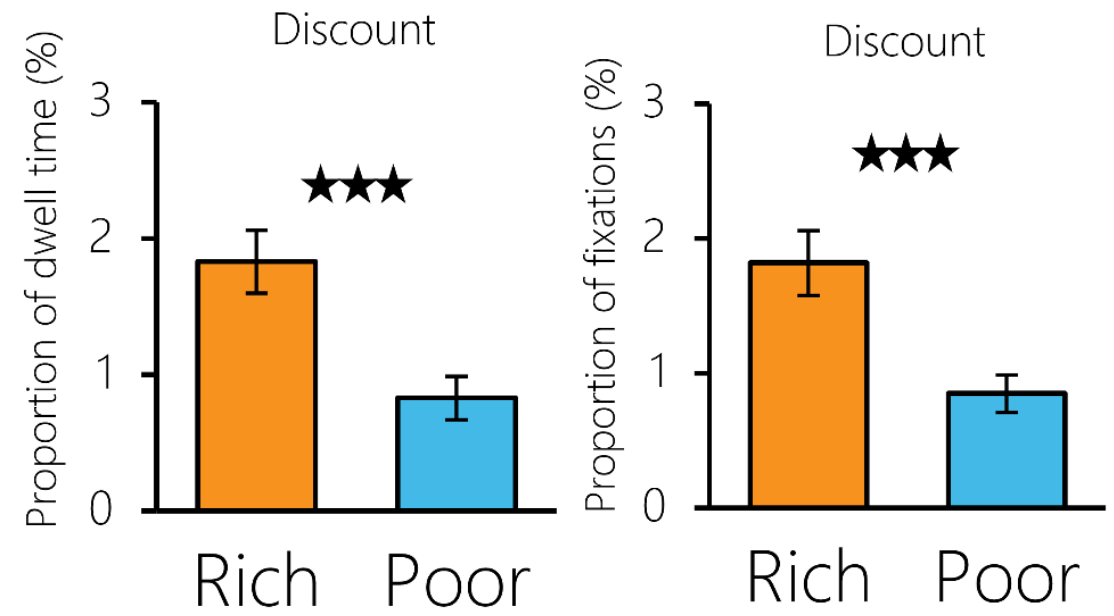

Fig. 2.5. The proportional dwell time and fixations on the discount clause between participants in the poor and the rich conditions (error bars reflect \pm 1 SEM; $* * * p<.001$ ).

An alternative explanation for the finding that the poor attended less to the discount was that scarcity might result in more efficient processing of task-relevant information. This would suggest that the poor did not need to look at the discount as much as the rich, because they were faster in seeing the discount. Since both the prices and the discount were task-relevant, this explanation would predict that the poor would be efficient in processing both price information and the discount. However, we found that the poor looked more at the prices but less at the discount than the rich did, which could not be explained by the efficiency account.

The discount on the menu could in theory help the poor participants save money and stay within their budget. Despite this usefulness, the poor participants still neglected the discount and 
focused more on the prices of the food items. This finding is ironic and could help explain why the low-income individuals engage in neglectful behaviors that are counter-productive. 
Chapter 3: Memory Enhancement of Scarcity-relevant Information

\subsection{Experiment 2}

Experiment 1 demonstrated that financial scarcity prioritizes the processing of price information, at the cost of other useful information. Given the attentional prioritization of prices, we predict that memory encoding of prices will also be enhanced. This prediction is supported by the recent work that suggests that visual working memory can be construed as visual attention preserved internally over time (Chun, 2011; Chun, Golumb, \& Turk-Browne, 2011). Featurebased theories of attention also predict selective facilitation in visual processing for task-relevant features (Hayden \& Gallant, 2009; Jehee, Brady, \& Tong, 2011). Thus, in Experiment 2 we examined the effects of scarcity on memory encoding, as a result of attentional prioritization. We predict that financial scarcity facilitates memory encoding specifically for price information, and not for other types of information.

\subsubsection{Participants}

A new group of 60 undergraduate students (43 female, 17 male; mean age $=19.95$ years, $S D=2.30$ ) from UBC participated in the experiment for course credit.

\subsubsection{Stimuli and Procedure}

To increase the demand for memory encoding, we increased the number of items on the menu. Participants were presented with a menu which now contained 50 food items. As in Experiment 1, the menu included the price and calories for each food item. Participants were asked to place a meal order from the menu as if they were ordering from a restaurant. There was no set time limit for participants to place their order. As before, participants were randomly 
assigned with a small budget ( $\$ 20$; the poor condition) or a large budget $(\$ 100$; the rich condition). The experiment again used a between-subjects design.

After participants placed their order, they were immediately given a surprise memory test. Participants were asked to recall as many items from the menu as possible. For each item recalled, they were also asked to recall the price and the calorie information of the item as accurately as possible. Participants typed the items, prices, and calories they could remember into a spreadsheet on a laptop computer that was provided to them.

\subsubsection{Results and Discussion}

To measure memory encoding, we calculated the average absolute error between the recalled prices (and calories) and the objective prices (and calories) for each participant (Figure 3.1). Participants in the poor condition (Mean error $=\$ 1.32$ ) were reliably more accurate in the price recall than those in the rich condition (Mean error $=\$ 2.19)[t(58)=2.42, p=.02, d=.63]$. However, there was no reliable difference in the calorie recall between the poor and the rich participants $[t(58)=.81, p=.42, d=.21]$.
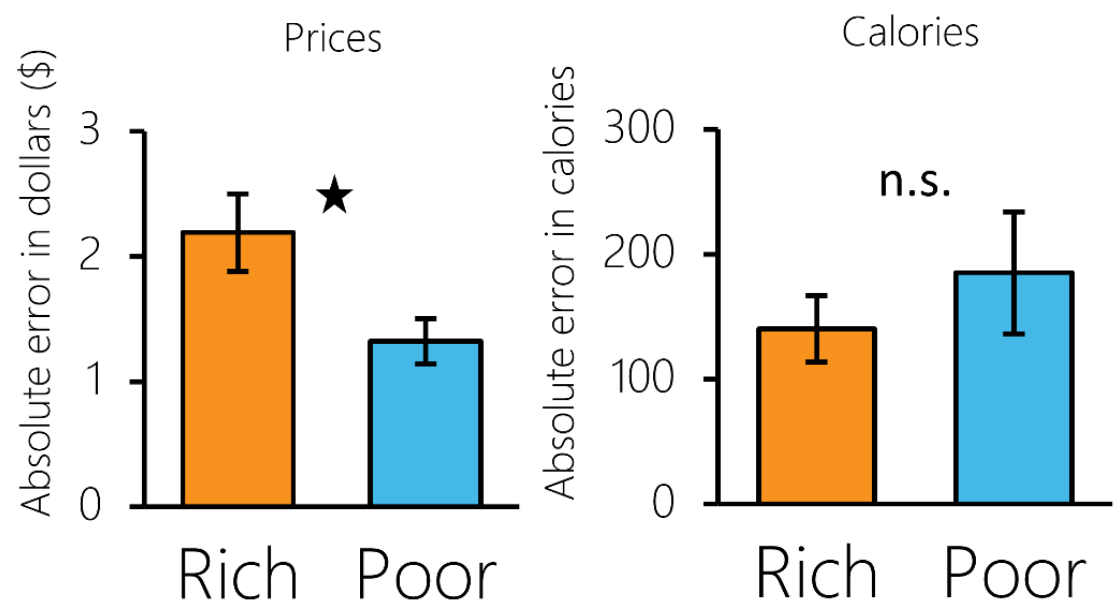

Fig. 3.1. The absolute error in the price recall and the calorie recall between participants in the price poor and the price rich conditions (error bars reflect $\pm l S E M$; ${ }^{*} p<.01$ ). 
This enhanced performance in price recall in the poor cannot be explained by the fact that the poor participants ordered fewer items $(M=2.13$ items $)$ than the rich $(M=3.70$ items $)[t(58)$ $=3.85, p<.001, d=.99]$. First, there was no reliable difference in the number of recalled times between the poor $(M=7.10$ items $)$ and the rich $(M=8.57$ items $)[t(58)=1.38, p=.17, d=.36]$. Second, there was no difference in the time taken to place the order between the poor $(M=73.55$ seconds) and the rich participants $(M=96.29$ seconds $)[t(58)=1.58, p=.12, d=.41]$. Third, even if ordering fewer items might improve memory recall, this benefit would be seen in both price and calorie recall, but we found that the poor were more accurate only in price recall, not in calorie recall. Thus, these findings suggest that financial scarcity improves memory encoding for task-relevant information (i.e., prices), but not for task-irrelevant information (i.e., calories). Scarcity selectively facilitates memory encoding.

\subsection{Experiment 3}

To generalize the findings in Experiment 2 to a different domain, we examined how calorie scarcity affects memory encoding. We predict that calorie scarcity facilitates memory encoding specifically for calorie information, and not for price information.

\subsubsection{Participants}

A new group of 60 undergraduate students (49 female, 11 male; mean age $=20.03$ years, $S D=2.11)$ from UBC participated in the experiment for course credit.

\subsubsection{Stimuli and Procedure}

The stimuli and the procedure were identical to those in Experiment 2, except for a critical difference. Participants were randomly assigned with a small calorie budget (500 calories; the poor condition) or a large calorie budget (2000 calories; the rich condition). As 
before, participants were then asked to place a meal order from the menu as if they were ordering from a restaurant. After the order, participants were given a surprise memory test, where they recalled items from the menu with the price and calorie information. Participants typed their responses into a spreadsheet on a laptop computer that was provided to them.

\subsubsection{Results and Discussion}

To measure memory encoding, we calculated the average absolute error between the recalled calories (and prices) and the objective calories (and prices) for each participant (Figure 3.2). Participants in the poor condition were reliably more accurate in the calorie recall (Mean error $=48.05$ calories) than those in the rich condition (Mean error $=71.61$ calories $)[\mathrm{t}(58)=$ $2.27, \mathrm{p}=.03, \mathrm{~d}=.58]$. This suggests that the calorie poor showed better memory encoding of calorie information than the calorie rich.
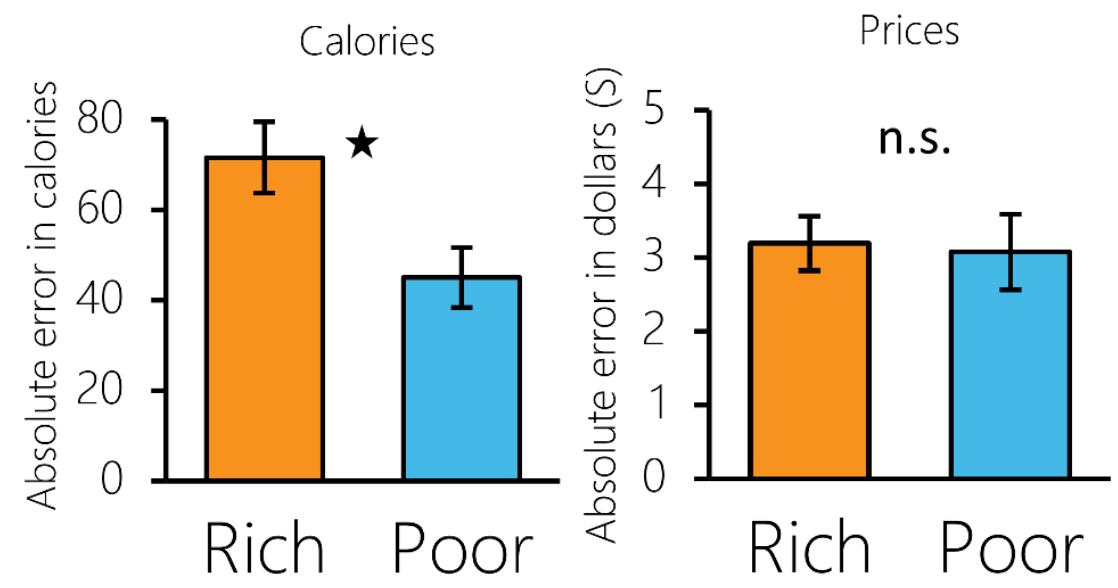

Fig. 3.2. The absolute error in the calorie recall and the price recall between participants in the calorie poor and the calorie rich conditions (error bars reflect \pm 1 SEM; *p<.01).

A critical test of our prediction was whether this memory facilitation is specific to taskrelevant information (i.e., calories). We found that there was no reliable difference in the price 
recall between the calorie poor $($ Mean error $=\$ 3.07)$ and calorie rich $($ Mean error $=\$ 3.19)$ conditions $[t(58)=0.19, p=.85, d=.04]$. Thus, memory encoding was selectively enhanced for the calorie information in the calorie poor participants. Interestingly, we did not observe worse memory encoding for the task-irrelevant information. That is, the calorie recall was the same for the price poor and the price rich in Experiment 2, and the price recall was the same for the calorie poor and the calorie rich in Experiment 3. The lack of difference between the rich and poor in memory encoding of task-irrelevant information could be driven by the possibility that neither the rich nor the poor needed to pay attention to this information in these experiments. 
Chapter 4: Spatial Narrowing of Attention under Scarcity

\subsection{Experiment 4}

In this experiment we investigated how time scarcity affects the online detection of information. Past work shows that people only start to increase their efforts to accomplish their goals when a deadline becomes salient (Gersick, 1988). Further, time pressure causes fewer attributes to be considered when choosing between alternatives (Wright, 1974). The goal of this experiment was to investigate how time scarcity affects the online detection of information in the environment. We hypothesize that time scarcity draws attention to the focal task, while inducing neglect of other useful information in the environment.

\subsubsection{Participants}

Undergraduate students $(N=90)$ were recruited from the UBC Human Subject Pool, and participated in the experiment in exchange for course credit.

\subsubsection{Stimuli and Procedure}

Each participant was asked to solve a series of puzzles on the computer screen. The puzzles were 50 trials of the Raven's Progressive Matrices (Raven, 2000). Each matrix appeared at the centre of the screen, and contained a pattern of objects. The bottom right corner of the matrix was missing, and participants had to find the right piece that fits with the general pattern in the matrix. The participant was asked to correctly solve as many matrices as possible in exchange for points. In each trial, participants were presented with one Raven's matrix, with the numbered pieces appearing below. The response keys appeared in a vertical list on the left side of the screen. In the top-left corner of the screen, the questions number and time remaining were 
displayed (see Figure 4.1). To solve a given matrix, participants had to press a number key that corresponds to the piece that fits into the missing corner of the matrix.

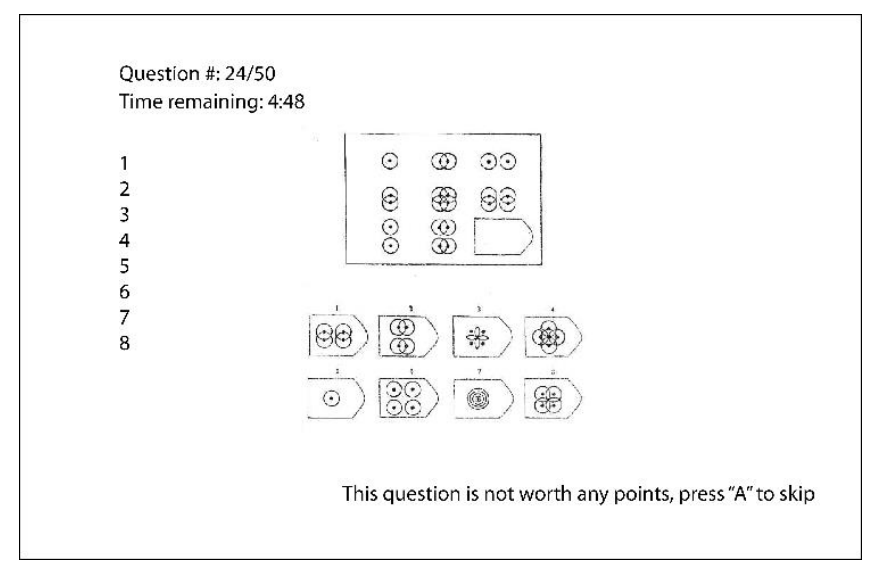

Fig. 4.1. Trial screen for Experiment 4.

To manipulate time scarcity, participants were randomly assigned with either a rich time budget (they had 40 minutes in total to solve the matrices; the time-rich condition, $N=45$ ), or a poor time budget (they only had 10 minutes in total to solve the matrices; the time-poor condition, $N=45$ ). Without explicit instruction or prompting, a time-saving cue appeared in the lower right part of the screen during the experiment. Specifically, on even-numbered trials starting from trial \#24, the cue appeared on the screen stating: "This question is not worth any points. Press 'A' to skip." (see Figure 4.1) Thus, 14 of the 50 trials were allowed to be skipped without any loss of points. The cue appeared at the same time as the matrix for those trials, and remained on the screen for $5000 \mathrm{~ms}$, and then disappeared. These trials presented an opportunity to skip the question in order to save time. Participants were not told anything about the cue. We wanted to see if they were able to detect this message during the experiment and skipped the even-numbered questions from trial \#24. 


\subsubsection{Results and Discussion}

Participants in the time-poor condition almost unanimously used their entire time budget while participants in the time-rich condition used less than half of their time budget. Given this constraint, the time-poor participants spent less time on the task overall $(M=9.54$ minutes $)$ compared to time-rich participants $(M=16.10$ minutes) $[t(88)=6.51, p<.001, d=1.37]$ (Figure 4.2a). The time-poor participants completed fewer trials $(M=46.02$ trials $)$ than the time-rich participants $(M=50$ trials) $[t(88)=4.71, p<.001, d=.99]$ (Figure $4.2 \mathrm{~b})$.

Notably, there was marginal difference in accuracy on the Raven's Progressive Matrices between the time-poor $($ Mean proportion correct $=.79)$ and the time-rich participants $($ Mean proportion correct $=.83)[t(88)=1.69, p=.09, d=.36]$ (Figure $4.2 c)$. When accounting for the total amount of time spent on the task, the time-poor participants scored higher accuracy per minute than time-rich participants $[t(88)=8.09, p<.001, d=1.71]$. This result suggests that time scarcity can cause a greater focus on the task at hand, enhancing task performance within the time limit.

Examining the number of questions skipped, we found that there was no difference in the average number of questions skipped between the time-poor $(M=2.51$ questions $)$ and the timerich participants $(M=3.78$ questions) $[t(88)=1.23, p=.22, d=.26]$ (Figure 4.2d). However, only $26.7 \%$ of the participants in the time-poor condition skipped at least once, and there were more time-rich participants $(48.9 \%)$ who skipped at least once $\left[X^{2}(1,90)=4.72, p=.03\right]$ (Figure 4.2e). This result suggests that time scarcity caused a failure to use the time-saving cue appearing on the bottom of the screen. Ironically, although the time-poor participants needed to use the time-saving cues more, they were less likely to take advantage of the time-saving opportunity than the time-rich participants. This illustrates how scarcity can create a vicious cycle: scarcity 
induces neglect of useful information in the environment, and as a consequence, the poor fail to save resources that can alleviate the condition of scarcity.
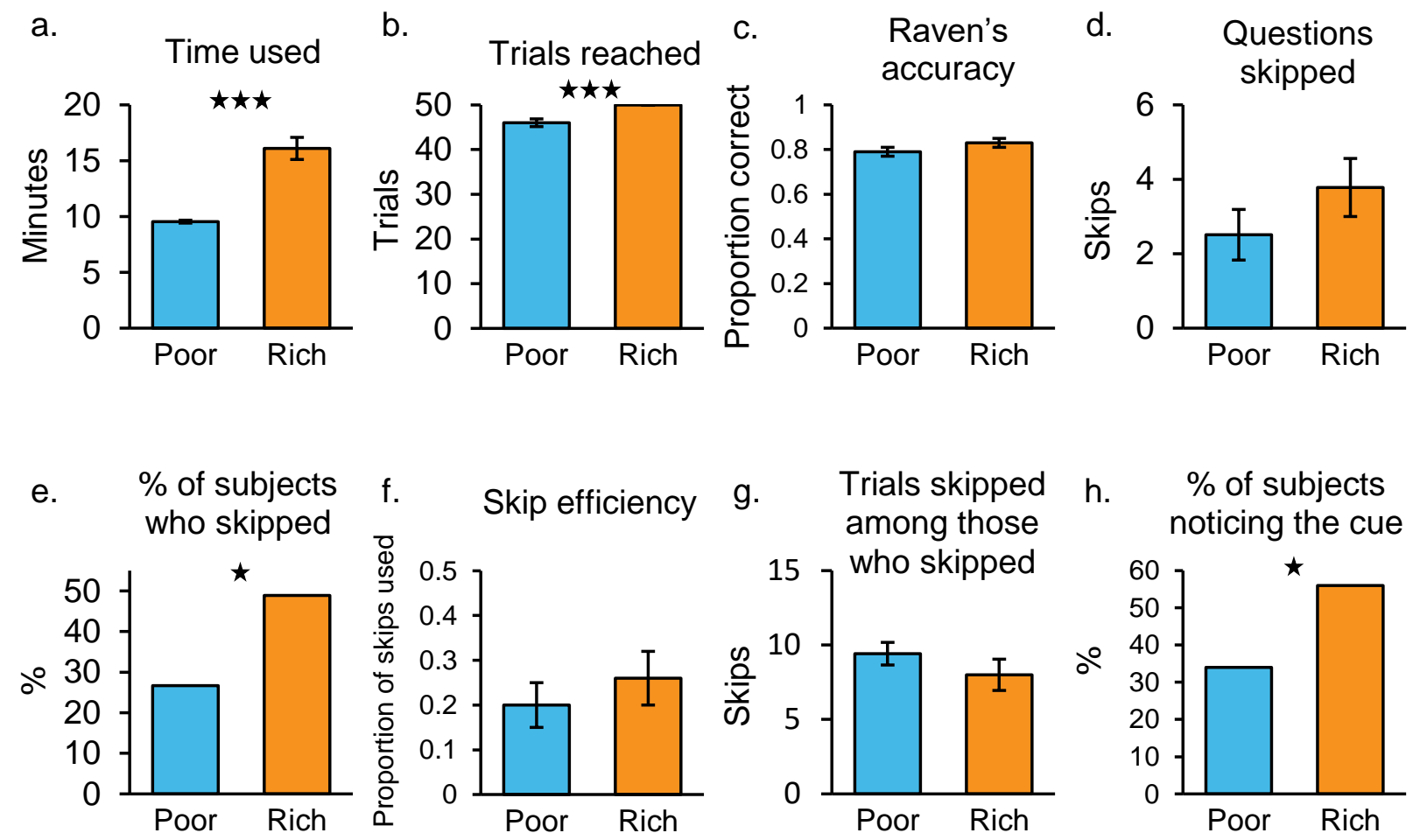

Fig. 4.2. Results for Experiment 4 (Error bars represent \pm 1 SEM. *p<.05, ***p<.001).

To control for the total number of trials completed, we calculated skip efficiency as the number of questions skipped divided by the number of possible questions that could be skipped. There was no difference in skip efficiency between the time-poor and the time-rich participants $[t(88)=.91, p=.36, d=.19]$ (Figure 4.2f)

Among those who skipped at least once, there was no difference in the number of questions skipped between the time-poor and the time-rich participants $[t(31)=.89, p=.38, d$ 
$=.34$ ] (Figure 4.2g). This means that if the participant noticed the cue at least once, they were able to skip the same number of questions, regardless of scarcity.

To measure retrospective recall of the time-saving cues, we asked participants after completing the task during debriefing to report whether they saw any messages appearing on the screen during the task. We found that the time-poor participants were less likely to report seeing the cues than the time-rich participants $\left[X^{2}(1,84)=3.81, \mathrm{p}=.05\right]$ (Figure 4.2h).

These results showed that fewer participants under time scarcity skipped the questions at least once, and reported seeing the cues, compared to time-rich participants. This suggests that time scarcity may narrow attention to the central task, while inducing a neglect of peripheral, even beneficial information in the environment. Alternatively, this finding may be explained by inattentional blindness, suggesting that the time-poor participants were less able to attend to salient but task-irrelevant information, than the time-rich participants. To tease these two accounts apart, we conducted the next experiment, probing whether scarcity alters the spatial scope of attention, or the ability to notice salient stimuli. Specifically, we manipulated the location of the time-saving cue, and examined the likelihood of skipping questions as a function of the spatial location of the cue under scarcity.

\subsection{Experiment 5}

In this experiment, we reduced the spatial distance between the time-saving cue and the matrix (i.e., the focal task) by moving the cue closer to the center of the screen, and investigated how the spatial proximity of the time-saving cue to the focal task impacted its detection.

\subsubsection{Participants, Stimuli, and Procedure}


Participants $(N=87)$ were recruited from the Human Subject Pool at UBC, and participated in the experiment in exchange for course credit. The stimuli and the procedure were exactly the same as those in Experiment 4, except one important change: the time-saving cue (i.e., the message to skip even-numbered questions after trial \#24) now appeared directly underneath the Raven's Matrix after trial \#24 for even-numbered questions (Figure 4.3).

If the neglect of the time-saving cue in Experiment 4 was due to the spatial narrowing of attention under scarcity, we would predict that the time-poor participants would be more likely to notice the cue if it were moved closer to the central task. On the other hand, if the neglect of the time-saving cue was due to inattentional blindness, moving the cue closer to the central task would not affect performance.

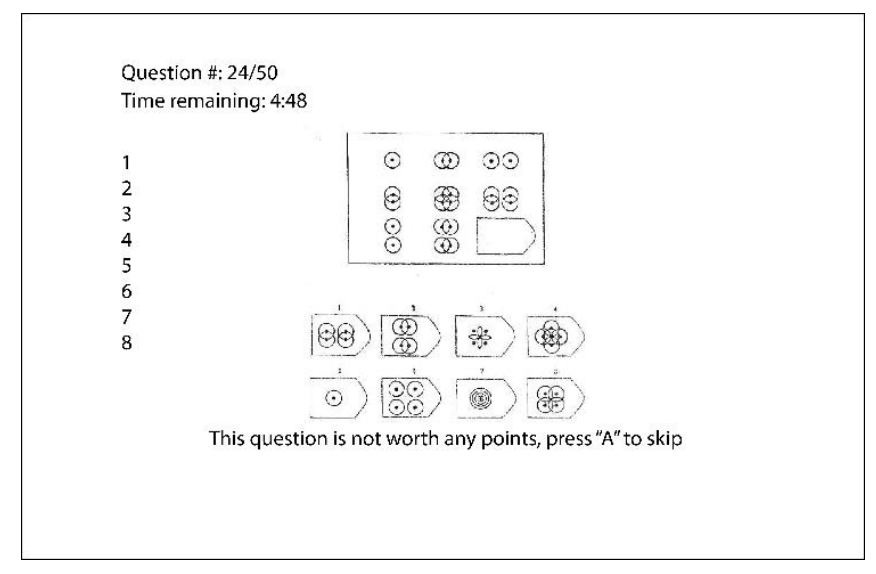

Fig. 4.3. Trial screen for Experiment 5, where the time-saving cue appeared right below the matrix.

\subsubsection{Results and Discussion}

Since in Experiment 4a, time scarcity influenced the number of participants who skipped at least once, we examined the same measure here again. We found that now there was no statistical difference in the percent of participants who skipped at least once $\left[X^{2}(1,87)=.56, p\right.$ $=.46]$. Comparing Figure $4.4 \mathrm{a}$ to Figure $4.2 \mathrm{e}$, the time-rich participants were not influenced by 
the change in the position of the cue, but the poor seemed to benefit from the closer proximity of the cue to the central task. This suggests that if the cue falls within the spatial scope of attention, the time-poor participants were able to take advantage of the cue.

During debriefing, the time-poor participants were marginally less likely to report seeing any messages during the task compared to the time-rich participants $\left[X^{2}(1,85)=3.583, \mathrm{p}=.06\right]$ (Figure 4.4b). Compared to the time-poor participants in Experiment 4 (34\% reported noticing the cue), the closer proximity seemed to provide a large benefit to the time-poor participants in Experiment 5 (49\% reported noticing the cue). These results support the account that scarcity narrows spatial attention to the focal task.

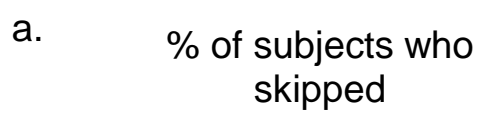
b. $\%$ of subjects noticing the cue
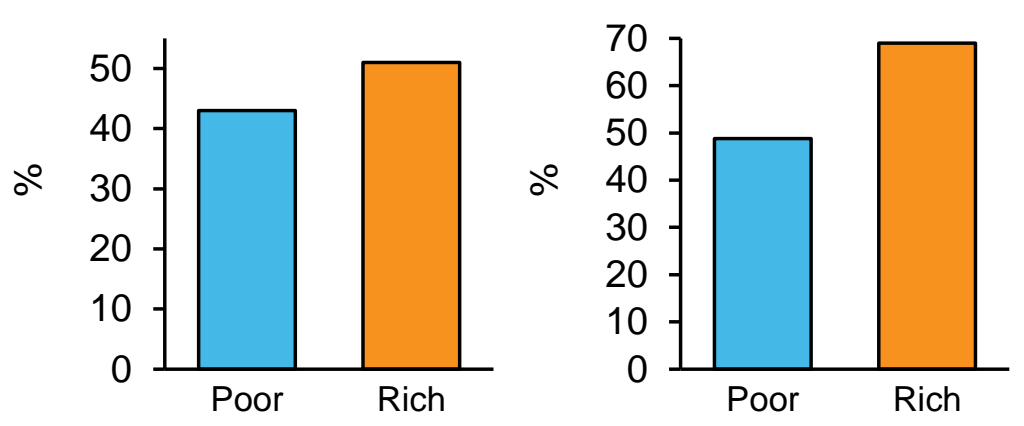

Fig. 4.4. Results for Experiment 5.

\subsection{Experiment 6}

To further explore the boundary condition of the spatial narrowing effect of scarcity, in this experiment we moved the time-saving cue farther away from the focal task, and examined how likely participants were to notice the cue. 


\subsubsection{Participants, Stimuli, and Procedure}

Participants $(N=86)$ were recruited from the Human Subject Pool at UBC, and participated in the experiment in exchange for course credit. The stimuli and the procedure were identical to those of Experiment 4, but this time the time-saving cue appeared in the bottom right corner of the screen (Figure 4.5), which was even farther away from the focal task than in Experiment 4.

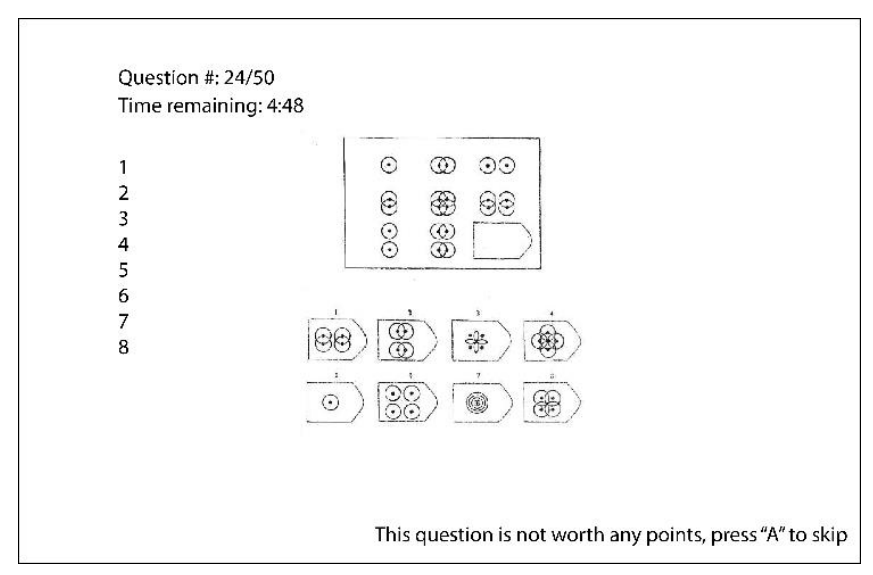

Fig. 4.5. Trial screen for Experiment 6, where the time-saving cue appeared far from the matrix, on the bottom right corner of the screen.

\subsubsection{Results and Discussion}

We found that participants in both conditions failed to take advantage of the cue. There was no difference in the percent of participants who skipped at least once $\left[X^{2}(1,86)=.93, p\right.$ $=.33$ ] (Figure 4.6a). During debriefing, there was no difference in the likelihood to report seeing any messages during the task between both conditions $\left[X^{2}(1,83)=2.00, p=.16\right]$ (Figure 4.6b). In fact, there was a floor effect in both the time-poor and the time-rich participants in skipping the questions or noticing the cue. This suggests that when the cue was spatially far away from the focal task, participants could not notice the cue, regardless of scarcity. 
a.

$$
\begin{aligned}
& \% \text { of subjects who } \\
& \text { skipped }
\end{aligned}
$$

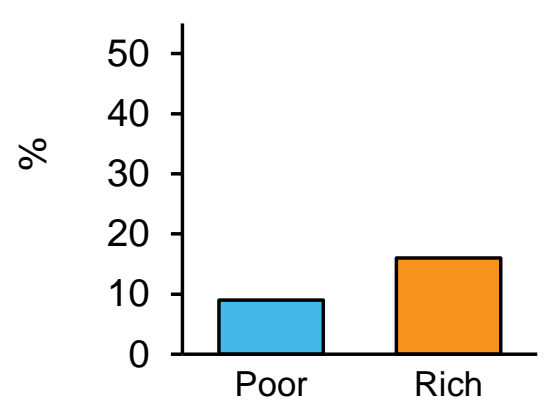

b.

$\%$ of subjects noticing the cue

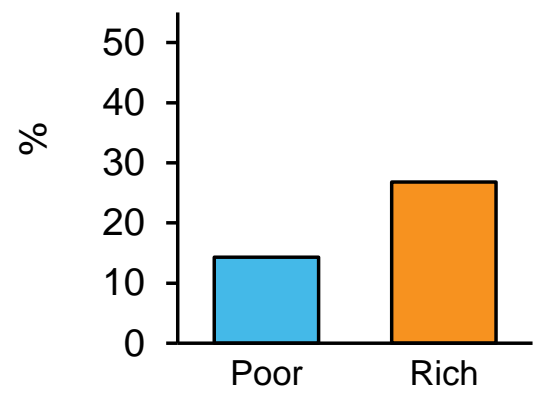

Fig. 4.6. Results for Experiment 6. 
Chapter 5: Prospective Memory Failures under Scarcity

\subsection{Experiment 7}

Experiments 4-6 showed that time scarcity narrowed attention on the focal task, resulting in the neglect of a time-saving cue which appeared in the peripheral during the experiment. However, in daily life, we do not always have cues in the external environment as reminders for certain actions. Instead, we need to rely on internal cues from memory that need to be activated at the right time to direct actions. For example, in order to pick up groceries on the way home from work, we must remember to turn at the right intersection in order to go to the grocery store. This depends on prospective memory, which is the ability to remember to execute future actions based on previous instructions. Cues for prospective memory are internal, and must be present in mind in order to cue behavior at the right time (Graf, Uttl, \& Dixon, 2002; Loftus, 1971). In this experiment, we examined how time scarcity affects prospective memory performance.

\subsubsection{Participants}

Participants $(N=90)$ were recruited from the Human Subject Pool at UBC and completed the study in exchange for course credit.

\subsubsection{Stimuli and Procedure}

Participants were asked to solve the same set of 50 Raven's Progressive Matrices used in Experiments 4-6. As before, participants were randomly assigned either a small time budget (5 minutes; the time-poor condition), or a large time budget (20 minutes; the time-rich condition). A critical difference in this experiment was that the time-saving cue never appeared in the experiment. Rather, all participants were explicitly instructed at the start of the experiment the following: "Even-numbered questions from number twenty-four on are not worth any points. 
You can skip these questions without losing any points." This instruction was presented on paper to participants to read, and the experimenter also read through these instructions with each participant to maximize the comprehension of the instruction. As before, the question number and remaining time appeared in the top-left corner of the screen, and the keys available for the participants to press were listed on the left side of the screen. Note that now the "A (skip)" key is listed among the available keys and was listed for every single question (Figure 5.1). There were no visual cues during the experiment to remind participants which questions they were allowed to skip. Thus, participants needed to remember to use the opportunity to skip when the applicable questions were reached.

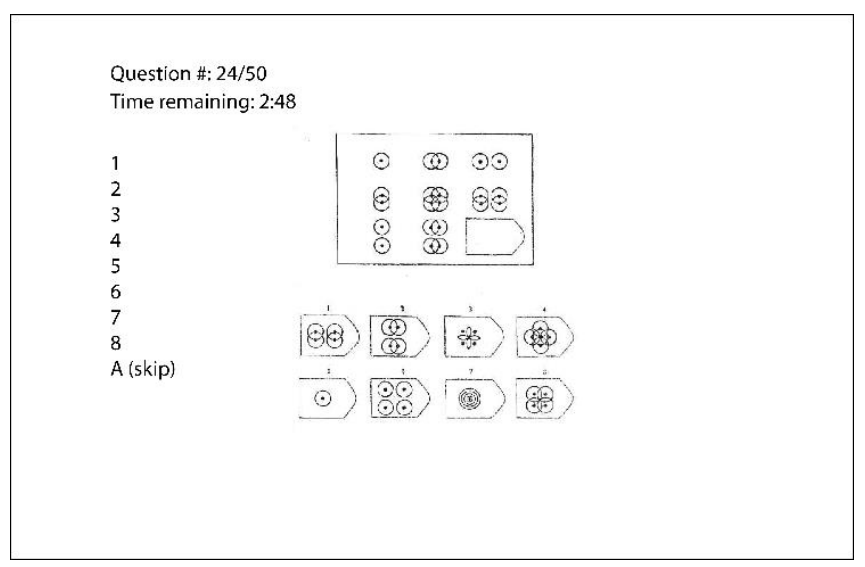

Fig. 5.1. Trial screen for Experiment 7.

\subsubsection{Results and Discussion}

Participants in the time-poor condition almost unanimously exhausted their time budgets, while participants in the time-rich condition usually completed the experiment with some time to spare (Figure 5.2a). The time-poor participants spent less time solving the Raven's Matrices ( $M$ $=4.88$ minutes $)$ than the time-rich participants $(M=14.03$ minutes $)[t(88)=13.33, p<.001, d=$ 2.81]. The time-poor participants also completed significantly fewer trials ( $M=35.40$ questions) than the time-rich participants $(\mathrm{M}=49.37$ questions) $[t(88)=10.14, p<.001, d=2.14]$ (Figure 
$5.2 \mathrm{~b}$ ), and were significantly less accurate (Mean proportion correct $=.76$ ) compared to the time-rich (Mean proportion correct $=.83)[t(88)=2.29, p=.02, d=.48]$ (Figure 5.2c). When accounting for the total amount of time spent on the task, the time-poor participants scored higher accuracy per minute than time-rich participants $[t(88)=9.53, p<.001, d=2.01]$, suggesting that time scarcity enhancing performance on the focal task.

The time-poor participants on average skipped fewer questions ( $M=2.51$ questions) than the time-rich participants ( $M=5.47$ questions) $[t(88)=2.52, p=.01, d=.53]$ (Figure 5.2d). However, this result is likely driven, at least in part, by the considerably smaller number of questions completed by the time-poor participants. Similarly, we found that fewer time-poor participants skipped at least once compared to the time-rich participants $\left[X^{2}(1,90)=10.08, p\right.$ $<.01]$ (Figure 5.2e), but this could be due to the smaller number of possible skips experienced by the time-poor participants. Thus, we examined the skip efficiency defined as the number of questions skipped divided by the number of possible questions that could be skipped experienced by the participant. We found that the time-poor participants were less likely to skip than timerich participants (two time-poor participants were excluded from this analysis due to failing to reach trial number twenty-four) $[t(86)=2.01, p=.05, d=.43]$ (Figure 5.2f). This finding suggests that time scarcity impairs prospective memory performance. We should note that among participants who skipped at least once, there was no difference in the number of questions skipped between the time-poor ( $M=7.5$ questions $)$ and the time-rich participants $(\mathrm{M}=8.6$ questions) $[t(40)=.59, p=.56, d=.19]$ (Figure 5.2g), or in skip efficiency $[t(40)=.76, p=.45$, $d=.26]$ (Figure 5.2h). 

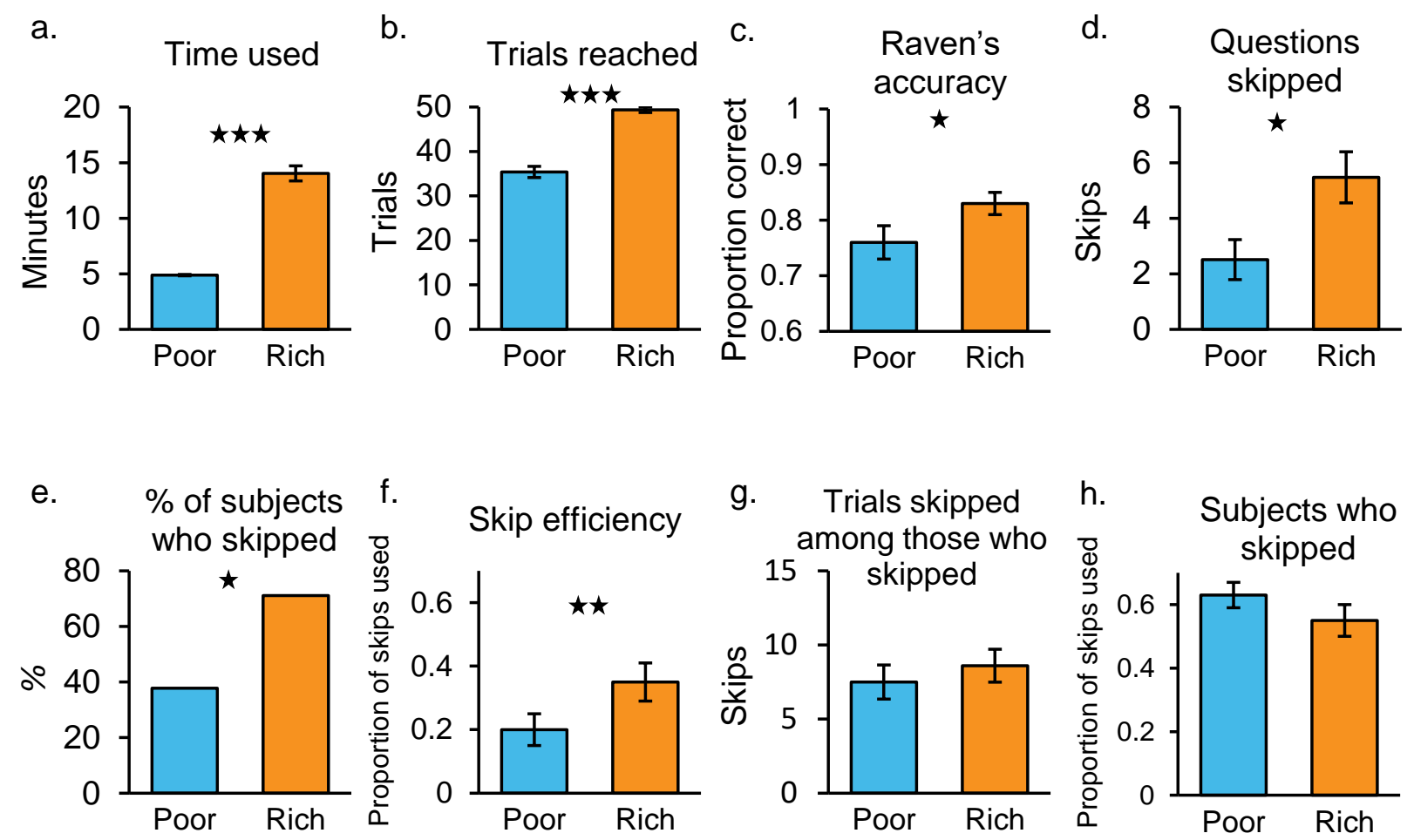

Fig. 5.2. Results for Experiment 7 (Error bars represent \pm 1 SEM. ${ }^{*} p<.05, * * p<.01, * * * p<.001$ ). 


\section{Chapter 6: Discussion}

The goal of the current study was to examine how scarcity forces attentional trade-offs and influences memory encoding driven by such trade-offs. When operating under a limited financial budget, the poor focused more on the price information, compared to the rich (Experiment 1). This focus came with the neglect of other information in the environment, even if the information could be useful or beneficial to the poor (e.g., the discount). The attentional prioritization of prices also resulted in enhanced memory encoding of price information among the poor participants (Experiment 2). Likewise, the attentional prioritization of calories led to better memory encoding of calorie information among the calorie poor (Experiment 3 ). We also found that people under time scarcity were less likely to take advantage of a time-saving cue that appeared peripheral to the focal task (Experiment 4), but nonetheless performed well on the focal task under the time constraint. This suggests that people under time scarcity are ironically less likely to notice opportunities to save time. This effect could be explained by a narrowing of spatial attention to the focal task (Experiments $5 \& 6)$. In the absence of an external cue, participants under time scarcity were less likely to remember to skip questions in the future (Experiment 7), suggesting that they failed to retrieve a cue from memory to execute actions at the right time.

These findings were particularly problematic for people under scarcity because the attentional neglect of resource-saving opportunities or the failure to remember to save resources could be detrimental, perpetuating the condition of scarcity and creating a vicious cycle of poverty. These cognitive impairments could explain a range of counter-productive behaviors observed in the low-income individuals, such as forgetting to follow instructions, or not signing up for public benefit programs. In addition, prospective memory errors can be seen by others as 
an indication of incompetence of the poor (Graf, 2012). The present findings instead attribute the memory failures not to the poor individuals themselves but to the condition of scarcity. The current study provides useful implications for designing policies and programs to mitigate the impact of scarcity, such as the use of reminders, automatic enrolment, or setting the right default, to reduce the attentional and memory burdens in the poor.

The current findings provide a new perspective on how scarcity shapes the way people perceive and experience the external environment. While the perceptual experiences can be largely characterized by information overload, scarcity selectively orients people's attention to specific aspects of the environment. When operating with financial constraints, people automatically prioritize price-relevant information. Such prioritization facilitates memory encoding of these information, but crucially it comes with a cost, which is the neglect of other information in the environment.

The current study also reveals a painful irony of scarcity. People with limited resources were too focused on prices, such that they neglected the beneficial discount that could save money and alleviate the financial burden. This irony can help explain why low-income individuals sometimes engage in neglectful behaviors that are counter-productive (e.g., missing an appointment for a health check-up, or failure to sign up for benefit programs).

It is worth noting that the current experiments involved an artificial simulation of scarcity in the lab. In fact, just by randomly assigning people to receive a hypothetical small or large budget, we observed a strong effect of scarcity on attention and memory. Moreover, the participants in our experiments were not provided with real money, were not rewarded for frugality, and knowingly were not to receive any food from the menu. In the absence of possible consequences of their decisions, the poor participants still focused on task-relevant information 
and neglected a help to alleviate the condition of scarcity. This raises the possibility that the present findings may not generalize to real-world resource scarcity. For example the cognitive system may up-regulate the perceptual salience of price discounts based on prior experience with financial scarcity. Alternatively, outside the lab, when people operate with scarce resources and can face real consequences of their actions, the effects of scarcity on attention and cognition observed in this study may be amplified. Forthcoming laboratory and field investigations should elaborate the cognitive effects and mechanisms of resource scarcity as it is experienced in the real world.

The current findings can help inform public policy and services targeting low-income populations. Among the OECD countries, enrollment in social assistance and public benefit programs is estimated to range between $40 \%$ and $80 \%$ (Hernanz, Malherbet, \& Pellizzari, 2004). Our current study provides a new explanation for the low participation rate. That is, the poor who are eligible for these programs fail to participate because of the attentional trade-offs under scarcity. Low-income individuals may need to focus on their financial challenges and deadlines under scarcity, and either are not aware of these benefit programs and services, or neglect the enrollment procedures. This attentional account is not the only factor that can explain the low participation rate. It is worth noting that in addition to the inherent cognitive and behavioural consequences that arise under the condition of scarcity, there are innumerable social stigmas and stereotypes associated with poverty. The low-income individuals are scorned, perceived as incompetent, and are disrespected (Fiske, 2011). People receiving food stamps are finger-printed to prevent cheating, and they undergo home visits to ensure they are actually living in poverty (Bertrand et al., 2004). Accordingly, the poor worry about being stereotyped as untrustworthy by other members in society. These stigmas of poverty can cause defensive responding in the poor 
and raise concerns about being judged according to the negative stereotypes, along with efforts to suppress negative thoughts and emotions in the service of self-regulation, which can consume executive resources (Schmader, Johns, \& Forbes, 2008) and disrupt cognitive performance (Spencer, Steele, \& Quinn, 1999). This effect, known as stereotype threat, can impair cognitive performance (Steele, 1997). In addition to these cognitive difficulties which must be navigated by the poor, the present study exemplified a new mechanism by which the poor are obstructed.

Given the attentional constraints under scarcity, we propose that social assistance and public benefit programs should be designed to avoid the attentional neglect in the poor under scarcity. The cognitive tax under scarcity may cause people to fail to remember sign-up deadlines, or to sign-up at all. New social services and programs should set up the right defaults, use automatic enrolment systems, and set up commitment devices. It may be helpful to streamline assistance applications and services to make them more salient, more accessible, and easier to process for the poor. The amount of effort and attention required from the poor should be minimized to increase or maintain participation. Benefit programs and social services can also be made more salient by using prompts and reminders. This could be done through any messaging medium such as text-message or email, and could be effective in catching the attention of those living under scarcity. Based on our current findings, future research can design behavioral interventions to avoid attentional neglect in the poor. 


\section{References}

Aarts, H., Dijksterhuis, A., \& Vries, P. (2001). On the psychology of drinking: Being thirsty and perceptually ready. British Journal of Psychology, 92(4), 631-642.

Baddeley, A. D., \& Hitch, G. J. (1974). Working memory. The psychology of learning and motivation, 8, 47-89.

Bernheim, B. D., Garrett, D. M., \& Maki, D. M. (2001). Education and saving: The long-term effects of high school financial curriculum mandates. Journal of Public Economics, $80(3), 435-465$.

Bertrand, M., Mullainathan, S., \& Shafir, E. (2004). Memos to the Council of BehavioralEconomics Advisors: A. In Behavioral-Economics View of Poverty. AEA Papers and Proceedings.

Chun, M. M. (2011). Visual working memory as visual attention sustained internally over time. Neuropsychologia, 49, 1407-1409.

Chun, M. M., Golomb, J. D., \& Turk-Browne, N. B. (2011). A taxonomy of external and internal attention. Annual review of psychology, 62, 73-101.

Fiske, S. (2011). Envy Up, Scorn Down: How Status Divides Us. NY: Russell Sage Foundation.

Gersick, C. J. (1988). Time and transition in work teams: Toward a new model of group development. Academy of Management Journal, 31(1), 9-41.

Graf, P. (2012). Prospective memory: Faulty brain, flaky person. Canadian Psychology/Psychologie Canadienne, 53(1), 7. 
Graf, P., Uttl, B., \& Dixon, R. (2002). Prospective and retrospective memory in adulthood. Lifespan Development of Human Memory, 257-282.

Gutierrez, H. C., \& Hershey, D. A. (2013). Impact of retirement worry on information processing. Journal of Neuroscience, Psychology, and Economics, 6, 264-277.

Hernanz, V., Malherbet, F., and Pellizzari, M. (2004). Take-Up of Welfare Benefits in OECD Countries: A Review of the Evidence. OECD Social, Employment and Migration Working Papers, 17, OECD Publishing, Paris.

Hayden, B. Y., \& Gallant, J. L. (2009). Combined effects of spatial and feature-based attention on responses of V4 neurons. Vision research, 49, 1182-1187.

International Energy Agency. (2016). World Energy Outlook 2016. Paris, Frances. Retrieved August 16, 2017 from: https://www.iea.org/newsroom/news/2016/november/worldenergy-outlook-2016.html

Jehee, J. F., Brady, D. K., \& Tong, F. (2011). Attention improves encoding of task-relevant features in the human visual cortex. The Journal of Neuroscience, 31, 8210-8219.

Loftus, E. F. (1971). Memory for intentions: The effect of presence of a cue and interpolated activity. Psychonomic Science, 23(4), 315-316.

Luck, S. J., \& Vogel, E. K. (1997). The capacity of visual working memory for features and conjunctions. Nature, 390, 279-281.

Mani, A., Mullainathan, S., Shafir, E., \& Zhao, J. (2013). Poverty impedes cognitive function. Science, 341, 976-980. 
Miller, G. A. (1956). The magical number seven, plus or minus two: Some limits on our capacity for processing information. Psychological Review, 63(2), 81.

Mullainathan, S., \& Shafir, E. (2013). Scarcity: Why having too little means so much. Henry Holt and Company, New York.

Pashler, H., Johnston, J. C., \& Ruthruff, E. (2001). Attention and performance. Annual Review of Psychology, 52, 629-651.

Piech, R. M., Pastorino, M. T., \& Zald, D. H. (2010). All I saw was the cake: Hunger effects on attentional capture by visual food cues. Appetite, 54, 579-582.

Radel, R., \& Clément-Guillotin, C. (2012). Evidence of motivational influences in early visual perception hunger modulates conscious access. Psychological science, 23(3), 232-234.

Raven, J. (2000). The Raven's progressive matrices: change and stability over culture and time. Cognitive Psychology, 41(1), 1-48.

Rock, I., \& Gutman, D. (1981). The effect of inattention on form perception. Journal of Experimental Psychology: Human Perception and Performance, 7, 275.

Salling, M., \& Harvey, M. E. (1981). Poverty, personality, and sensitivity to residential stressors. Environment and Behavior, 13(2), 131-163.

Schmader, T., Johns, M., \& Forbes, C. (2008). An integrated process model of stereotype threat effects on performance. Psychological Review, 115, 336-356.

Shah, A. K., Mullainathan, S., \& Shafir, E. (2012). Some consequences of having too little. Science, 338, 682-685. 
Shah, A. K., Shafir, E., \& Mullainathan, S. (2015). Scarcity frames value. Psychological Science, 26(4), 402-412.

Shapiro, G. K., \& Burchell, B. J. (2012). Measuring financial anxiety. Journal of Neuroscience, Psychology, and Economics, 5, 92-103.

Simons, D. J., \& Chabris, C. F. (1999). Gorillas in our midst: Sustained inattentional blindness for dynamic events. Perception, 28, 1059-1074.

Spencer, S. J., Steele C. M., \& Quinn, D. M. (1999). Stereotype threat and women's math Performance. Journal of Experimental Social Psychology, 35, 4-28.

Steele, C. M. (1997). A threat in the air: How stereotypes shape intellectual identity and performance. American psychologist, 52(6), 613.

Stetter, F., Ackermann, K., Bizer, A., Straube, E. R., \& Mann, K. (1995). Effects of diseaserelated cues in alcoholic inpatients: results of a controlled "alcohol Stroop" study. Alcoholism: Clinical and Experimental Research, 19, 593-599.

Strayer, D. L., Drews, F. A., \& Johnston, W. A. (2003). Cell phone-induced failures of visual attention during simulated driving. Journal of Experimental Psychology: Applied, 9, 2332.

Tomm, B. M., \& Zhao, J. (in press) Scarcity impairs online detection and prospective memory. Proceedings of the $39^{\text {th }}$ Annual Conference of the Cognitive Science Society. Austin, TX: Cognitive Science Society.

Tomm, B. M., \& Zhao, J. (2016). Scarcity captures attention and induces neglect: Eyetracking and behavioral evidence. In A. Papafragou, D. Grodner, D. Mirman, \& J.C. Trueswell 
(Eds.), Proceedings of the 38th Annual Conference of the Cognitive Science Society (pp. 1199-1204). Austin, TX: Cognitive Science Society.

UNICEF and World Health Organization. (2015) Progress on Sanitation and Drinking Water: 2015 update and MDG assessment. Geneva, Switzerland. Retrieved August 16, 2017 from: http://www.who.int/water_sanitation_health/monitoring/jmp-2015-update/en

World Bank (2016). Poverty and Shared Prosperity 2016: Taking on Inequality. Washington, DC: World Bank. doi:10.1596/978-1-4648-0958-3.

Wright, P. (1974). The harassed decision maker: Time pressures, distractions, and the use of evidence. Journal of Applied Psychology, 59(5), 555-561.

Zhao, J., \& Tomm, B. M. (2017, July). Attentional Trade-Offs Under Resource Scarcity. In International Conference on Augmented Cognition (pp. 78-97). Springer, Cham.

Zhao, J., \& Tomm, B. M. (under review) Psychological responses to scarcity. Oxford Research Encyclopedia of Psychology. DOI: 10.1093/acrefore/9780190236557.013.41. 